OCTOBER 1987

LRP 330/87

DYNAMICAL PLASMA RESPONSE TO ADDITIONAL HEATING

B. Joye, J.B. Lister, J.-M. Moret, A. Pochelon, C.W. Simm 


\title{
DYNAMICAL PLASMA RESPONSE TO ADDITIONAL HEATING
}

\author{
B. Joye, J.B. Lister, J.-M. Moret, A. Pochelon and C.W. Simm
}

Centre de Recherches en Physique des Plasmas
Association Euratom - Confédération Suisse
Ecole Polytechnique Fédérale de Lausanne
21, Av. des Bains, CH-1007 Lausanne, Switzerland

\begin{abstract}
The response of a tokamak plasma to an additional heating pulse is enigmatic. This paper presents results of the dynamical response on the electron temperature, deduced from the soft X-ray emissivity profile, to additional Alfvén Wave Heating Power. Most of this study used quasi steady state sinusoidal modulation of the delivered RF power, but data are also included on the step-function response at the end of the RF pulse, and the impulse response to short periodic RF pulses. These different approaches all show similar behaviour of the radial profile of the response phase, which is dominated by a central flat region within the $q=1$ surface, with, at the higher frequencies used, a minimum phase of close to $180^{\circ}$. Moving further outside the $q=1$ radius the phase increases extremely sharply. This basic character of the phase profile is not altered by varying the calculated power deposition profile but is still defined by the $\mathrm{q}=1$ radius, demonstrated by a plasma current scan at constant toroidal field. In addition to the soft X-ray flux baseline modulation, the sawtooth period is modulated, but out of phase with the RF power. These effects have been studied as a function of modulation frequency, plasma current, RF power and Alfvén Wave mode.
\end{abstract}




\section{INTRODUCTION}

The response of tokamak plasmas to additional heating remains extremely confusing, especially the reticence towards strongly modifying the electron temperature profile. When Alfvén Wave Heating (AWH) is applied to the TCA tokamak, a highly structured response is seen on the soft $\mathrm{X}$-ray emissivity profile, an example of which is shown in Fig. 1. The evolution observed has so far defied a simple explanation in terms of the radial positions of the AW resonance layers. The average soft X-ray fluxes, the sawtooth amplitude and the sawtooth period all change. Even at the RF turn off there is no obviously simple pattern to the flux profile evolution. A more systematic technique in

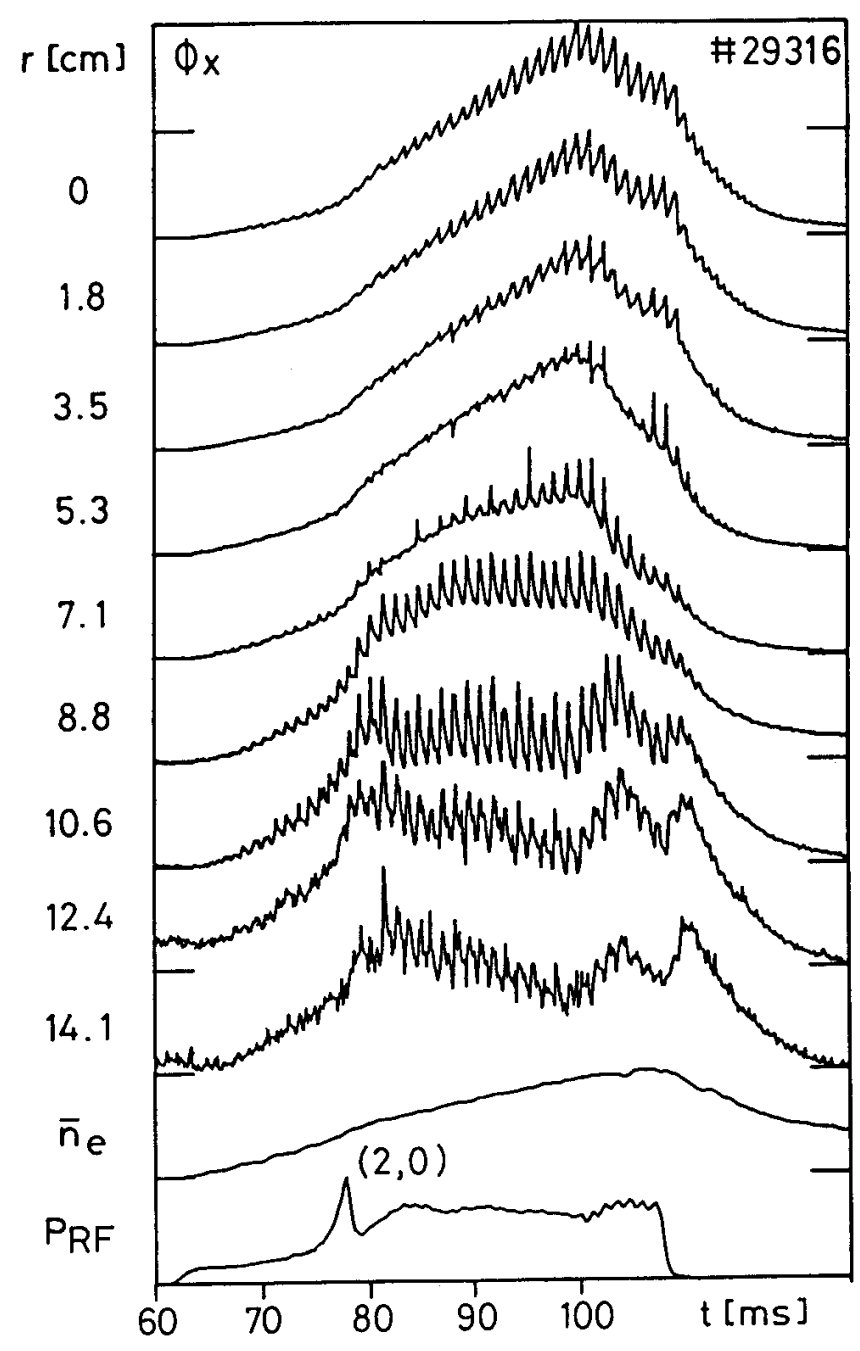

Fig. 1 Typical soft X-ray flux traces obtained during an $A W H$ pulse, illustrating the non-uniform response $[2.5 \mathrm{MHz}, 120 \mathrm{kA},(N, M)=(2,1)]$. 
such cases is to study the harmonic response, and this paper presents results using this approach. Preliminary results in limited conditions have already been reported on D-III (JAHNS et al., 1986), on W-VIIA (HARTFUSS et al., 1986) and on JET (JET TEAM, 1987). The first two used square-wave modulated electron cyclotron heating, and the last sinusoidally modulated ICRF power. RF power sources have been equipped for such experiments only in the last few years. In principle, the response spectra should yield both the additional heating power deposition profile and the thermal conductivity profile, provided that the modulating frequency range is correctly chosen with respect to the electron energy confinement time $\left(1 / \omega \approx \tau_{\mathrm{Ee}}\right)$, provided that the energy absorption is local and direct, and provided that the heat transport is dominated by a local, linear, diffusive process. Our results will question the correctness of the latter provisions.

In Section 2 of this paper, the experimental procedures and the analysis techniques are described. Section 3 presents the data obtained from an analysis of the harmonic component of the soft X-ray signals and the response to different transients. Modulating the RF power also produces a harmonic modulation of the sawtooth period, discussed in Section 4. The implications of the results are discussed in Section 5, followed by a summary in Section 6.

\section{EXPERIMENT AND METHOD}

The experiments described were carried out on the TCA tokamak $(\mathrm{R}, \mathrm{a}=0.61,0.18 \mathrm{~m})$ with the following conditions : $\mathrm{B}_{\phi}=1.51 \mathrm{~T}, \mathrm{I}_{\mathrm{p}}<130 \mathrm{kA}, \mathrm{n}_{\mathrm{e}}<6 \cdot 10^{19} \mathrm{~m}^{-3}$; the filling gas was deuterium. The additional heating was provided by Alfvén Waves launched by 8 phase-coherent antennae. The TCA tokamak has been described in detail by CHEETHAM et al. (1981) and the Alfvén Wave excitation and heating experiments by COLLINS et al. (1986) and BESSON et al. (1986). The RF excitation frequency was 2.0 or $2.5 \mathrm{MHz}$, and the delivered RF power was less than $100 \mathrm{~kW}$ to obtain a quasi steady state within the RF pulse, which lasted up to $80 \mathrm{msec}$, during the plasma current flat-top. This delivered power is to be compared with approximately $200 \mathrm{~kW}$ of ohmic heating power. The RF generator is a linear amplifier chain, allowing us to modulate the first, 
low power, pilot stage, to achieve high power modulation.

A specific problem for modulation using Alfven Wave Heating is that the excited modes, and the positions of the continuum resonance layers, vary with the plasma density. Since the density increases strongly when the RF power is applied, we have so far been limited in the amount of RF power delivered while retaining a quasi steady state. On the other hand, varying the wave excitation conditions within one single discharge should lead to an extremely sensitive measurement of any sudden change in the response.

The major diagnostic for the experiments described was a 15 channel soft X-ray pin-hole camera viewing the plasma horizontally. This system has a channel spacing of about $18 \mathrm{~mm}$, an equivalent channel width of $4-18 \mathrm{~mm}$ (centre-edge), and an adequate time resolution. The signals were filtered and acquired with an 80-160 $\mu$ sec sampling interval, compared with the $0.5-2$ msec sawtooth period. Although the modulated RF power produces a modulated response of most plasma parameters, the only other diagnostic studied in detail so far was the 8-channel FIR $(447 \mu \mathrm{m})$ interferometer which was used to confirm that the electron temperature modulation dominates the modulated soft X-ray response, as will be discussed.

The experimental conditions which we systematically varied were the frequency of the RF modulation (mostly in the range $30-500 \mathrm{~Hz})$, the modulation depth $(0-90 \%$ peakpeak/max.), the average RF power level and the plasma current over the range 55-130kA. The Alfvén Wave excitation mode was mainly $(N, M)=(2,1)$ where $(N, M)$ are the dominant imposed toroidal and poloidal mode numbers, but was also changed to $(\mathrm{N}, \mathrm{M})=(4,1)$ to search for an effect of the expected change in the power deposition profile.

The major part of both the experimental and analysis effort was devoted to the sinusoidal modulation experiments. The extraction of the modulated part of the experimental traces is delicate, when the pulse conditions drift (not a quasi steady state), when the response might not be constant as a function of time, and, above all, when the number of cycles is limited by the requirement for some degree of time resolution of the 
measured dynamical response. We must also consider that the RF power modulation is not purely sinusoidal, and estimate the complex amplitude of the modulation of the driving function, the RF power, as well as that of the plasma diagnostic signals. We can then determine the relative phase and the response gain, respectively expressed as the opposite argument (a positive phase refers to a delay) and modulus of the complex ratio of the two modulation amplitudes. In what follows we shall refer to $\mathbf{A}(v)$ as the frequency dependence, for example, of any complex amplitude $\mathbf{A}$, with its phase defined relative to the driving function. The notation $A^{*}$ refers to the normalized response equal to $(\mathbf{A} /<\mathrm{A}>) / \mathbf{P}_{\text {rf. }}$

For quasi steady state conditions we could simply perform a Fourier Transform of the two signals, but this analysis proved to be inadequate for handling the shorter periods and slewing signals. The method of cross-power analysis corresponds to a similar treatment, used by JAHNS et al. (1986) and HARTFUSS et al. (1986). We have found that an improved procedure is to perform a true fit to the data using the least-squares criterion, to a specified waveform $y\left(t^{\prime}\right)$ given by

$$
\mathrm{y}\left(\mathrm{t}^{\prime}\right)=\operatorname{Re}(\mathbf{Y}(\mathrm{t})) \cdot \cos \left(\omega \mathrm{t}^{\prime}\right)+\operatorname{Im}(\mathbf{Y}(\mathrm{t})) \cdot \sin \left(\omega \mathrm{t}^{\prime}\right)+\langle\mathrm{y}\rangle(\mathrm{t})+\frac{\mathrm{d}<\mathrm{y}\rangle}{\mathrm{dt}}(\mathrm{t}) \cdot\left(\mathrm{t}-\mathrm{t}^{\prime}\right)
$$

The coefficients corresponding to time $t$ are considered valid for $\left|t-t^{\prime}\right|<\Delta T / 2$ where the fit window has a duration $\Delta \mathrm{T} . \mathrm{Y}(\mathrm{t})$ is the complex modulation amplitude to be estimated, $v=\omega / 2 \pi$ is the modulation frequency, $\langle y\rangle(t)+d<y\rangle / d t \cdot\left(t^{\prime}-t\right)$ is the linear unmodulated drift plus offset of the background signal.

Using a full 4-variable fit allows us to treat any time interval, which is not the case if we separate the linear background and then analyse the residual, especially if the window contains a non-integral number of periods. This is easily seen on test data. We have only used a square weighted fitting window up to now. A second advantage to this fit is that its linear form allows an efficient fitting procedure to be used. An estimate for the error on the fitted values is then simply obtained from the fit covariance matrix, 
assuming standard distributions of the deviations. This procedure is quite expensive in computation, but we consider that it yields truer estimators during transient evolutions, than the simpler "filter-and-analyse" procedure.

Typically we were able to use $40 \mathrm{msec}$ of steady state modulation to obtain the asymptotic response with minimal error. To follow the time-variation of the response amplitude and phase we reduced the window to 5-10 msec corresponding to $0.5-20$ modulation cycles. The resulting error depends more on the analysis time window than on the number of cycles, due to the reduction in the modulation amplitude with increasing frequency. The RF power modulation experiments give very accurate and reproducible amplitude and phase profiles since the response is taken to be sinusoidal, and can be sampled over an arbitrarily long time, reducing the noise contribution.

On the other hand, the experiments which looked for a transient response or a step response were analysed using the relative time delays of some distinctive, but otherwise fairly arbitrary feature of the experimental traces in order to obtain a radial delay curve. These data are included in this paper due to the striking similarities of some of the experimental features using both techniques.

The analysis of the sawtooth period $\tau_{\mathrm{ST}}$ is delicate due to the presence of the $\mathrm{m}=1$ oscillations and the change in the shape of the sawtooth waveform during the RF pulse. We adopted a fairly simple definition, timing the zero-crossings of the high-pass filtered data. This analysis was adequate for the large changes in period which were measured. Although modulating the sawtooth amplitude, but not the baseline, will lead to a non-zero Fourier component, as seen on JET by GAMBIER (1987), our results show such a large amplitude of the baseline modulation, that we do not need to separately treat the sawtooth crash amplitude. Samples of data analysed by taking the end of each crash as the base line, showed an insignificant change in the response phase or amplitude.

We finish this section by discussing the significance of the results derived from the soft X-ray signals. The main advantages are that the spatial and temporal resolutions are perfectly suited to measure smooth values of the energy deposition and transport profile. 
The analysis of the global magnetic signals $\left(\beta_{\mathrm{dia}}\right.$ or $\left.\beta+1_{\mathrm{i}} / 2\right)$ is complicated by the shell time which corresponds to the frequency range explored, and leads to a $45^{\circ}$ phase error even at the low frequency of $100 \mathrm{~Hz}$.

The disadvantage is that the soft X-ray flux after passing the beryllium filter is not a direct measurement of electron temperature, but a line-integral of the emissivity $\phi_{\mathbf{X}}(r) \approx$ $n_{e}{ }^{2}(r) \cdot T_{e}(r)^{\alpha}\left(T_{e}\right) \cdot f\left(Z_{e f f}, Z_{i}\right)$. The radial profile is, however, extremely peaked, allowing us to forgo an Abel inversion in the standard analysis; the conclusions are not altered by such an additional treatment. The following problems do, however, remain :

(i) Since we are viewing horizontally, radial deformation of the equilibrium will modulate the signal seen by the pin-hole arrangement. We estimate this correction to be negligible at the $10 \%$ level with respect to the observed signal modulation;

(ii) The flux depends on the plasma density which is itself modulated by the RF power. Figure 2 gives the phase and amplitude of the density fluctuations, obtained by Abel inverting the projected profile before fitting to the modulated waveform; the density modulation is small, typically less than $1 \%$ above $100 \mathrm{~Hz}$ in the central region of the plasma, where it has no effect on the soft X-ray modulation; towards the edge, the relative density modulation reaches 3-4\%, where it causes a 6-8 \% modulation in the soft $\mathrm{X}$-ray flux, compared with $60 \%$ modulation observed; the phase starts near $0^{\circ}$ at the plasma edge and rises to $180^{\circ}$ in the centre at $150 \mathrm{~Hz}$;

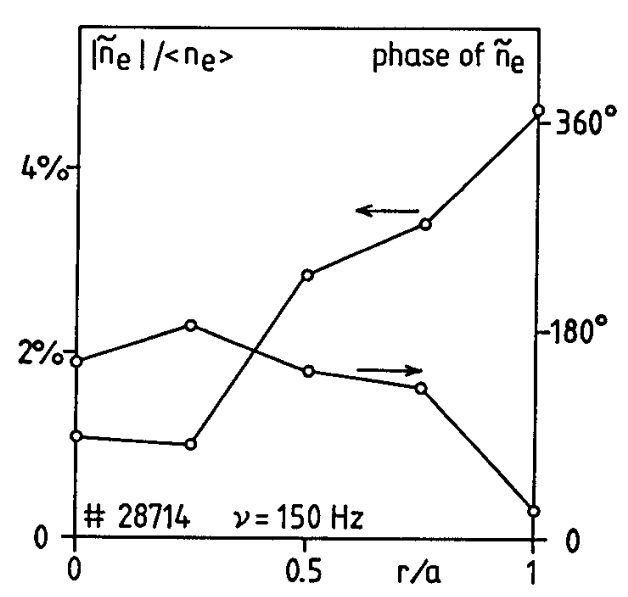

Fig. 2 Modulation of the electron density profile [2.5MHz, $126 \mathrm{kA},(N, M)=(2,1),\left\langle P_{r f}\right\rangle \approx 60 \mathrm{~kW}, / P$ rf $/ \approx 40 \mathrm{kw}$, $v=150 \mathrm{~Hz}]$ 
(iii) A modulation of the impurity contribution is in principle possible by modulating the electron temperature, thereby changing the charge state equilibrium, or by modulating the impurity influx. We do not consider that either of these effects is large enough to significantly perturb our results. The bolometer sees very little modulation of the radiated power;

(iv) The variable dependence on the electron temperature as a function of radius $\left(\alpha\left(T_{e}\right)\right.$ varies from the center to the edge from say $2-4$ for $\left.25 \mu \mathrm{m} \mathrm{Be}\right)$, prohibits us from evaluating the radial profile of $T_{e}(r) /<T_{e}(r)>$. However, the phase of the modulation is unaffected, and none of the following discussion requires a calibration of the temperature modulation.

As a result of these arguments, we take the modulation of the soft X-ray signals to be due to an electron temperature modulation, as did JAHNS et al. (1986).

\section{SOFT X-RAY DYNAMICAL RESPONSE}

In the following sub-sections we firstly present the raw soft X-ray data from a typical RF power modulation experiment. Then the radial profile of the response is looked at as a function of the modulation frequency; links are made to results for step response and impulse response data. The excited Alfvén Wave mode is then changed to vary the theoretically calculated power deposition profile. A plasma current scan at fixed toroidal field explores the effect of $q_{a}$, both for the harmonic response and the step response. Finally we present data on the dependence on the RF power level used, and the time-dependence of the response gain. The implications of the data presented in this section are discussed later in Section 5. All the data in this present section refer to the signals from which the sawteeth have effectively been filtered out by using the imposed harmonic fit as described in Section 2. 


\subsection{Raw experimental data}

Two typical modulated RF discharges are shown in Fig. 3(a,b). In the first case the RF power is modulated throughout the RF pulse; in the second, the modulation is gated on for the last $30 \mathrm{msec}$ of the RF pulse. Both discharges are modulated at a frequency of $150 \mathrm{~Hz}$ with a modulation amplitude of $30 \mathrm{~kW}$.

The three soft $\mathrm{X}$-ray channels shown in the figure, $\mathrm{r}=0.0,0.071,0.141 \mathrm{~m}$ are all modulated to a depth considerably greater than the sawtooth crash variation. The harmonic fit is not, therefore, simply picking up the sawtooth amplitude and period modulation, which is also illustrated in Fig. 3.

The signal modulation, and the line-averaged density, reach a steady state for the last $30 \mathrm{msec}$ in both cases, and this full window was used for the most accurate determination of amplitude and phase, as discussed in Section 2. Both modulation response phase and amplitude are very strong functions of the plasma minor radius. At this low modulation frequency, the amplitude and phase are easily gauged by eye, although at higher frequency the fitting procedure yields parameters which are not visible. In the following we discuss the coefficients obtained from the 4-parameter fit.

\subsection{Frequency variation}

The frequency of modulation was varied from $v=20-500 \mathrm{~Hz}$ with a roughly constant power modulation depth. Figure 4 shows the amplitude and phase of the response as a function of the modulation frequency from $100-300 \mathrm{~Hz}$ for three diodes, $\mathrm{r}=0.0,0.071,0.106 \mathrm{~m}$. The phase of the outer channel $(\mathrm{r} / \mathrm{a} \approx 0.6)$ is large at high frequency, close to $360^{\circ}$, which could be confused with a prompt response with zero delay. The phase as a function of frequency must be continuous from $0^{\circ}$ at zero frequency, which requires the apparent $2 \pi$ ambiguity to be resolved as a true $360^{\circ}$ phase. 
This is also clear from the gated modulation case, Fig. 3(b), in which the outer diode is fully modulated after nearly one full cycle, and remains modulated even after the RF turnoff, again for close to a full cycle.

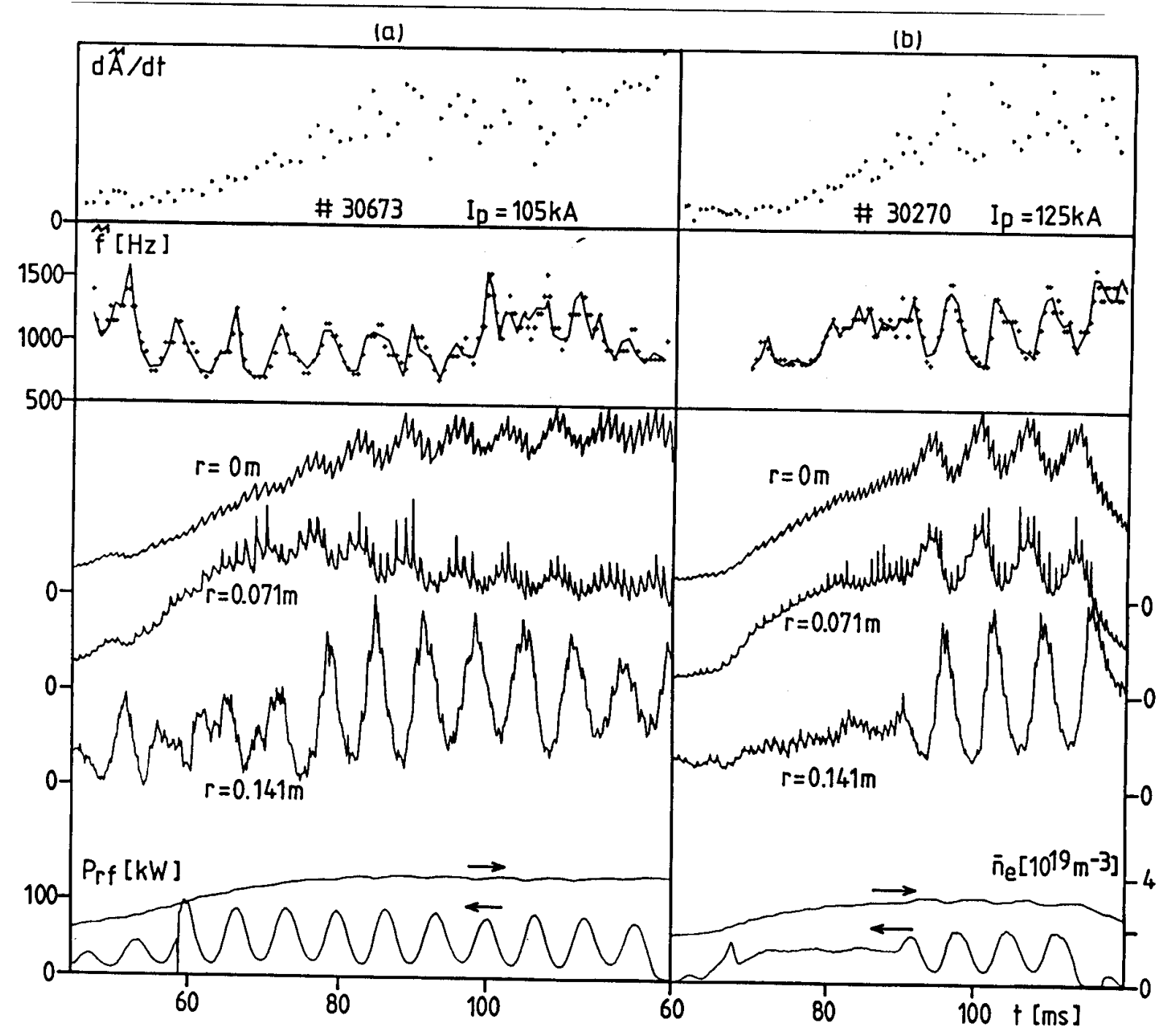

Fig. 3 Schematic of typical traces during the RF power modulation at $150 \mathrm{~Hz}: d A S T / d t ; f S T ; A X(r=0.0,0.071$, $0.141 \mathrm{~m}$ ); $n_{e}$ and $P_{r f}$. Two discharges are shown : (a) \#30673 [2.0MHz, 105kA] is modulated throughout the RF pulse and (b) the modulation is gated on for the later part of the pulse for \#30270 [2.5MHz, $125 k A]$.

The amplitude dependence on frequency, $\left|A^{*}(v)\right|$ shows a roughly $1 / v$ dependence on all three diodes, as we might expect for an integrating system relating $d T_{\mathrm{e}} / \mathrm{dt}$ to $\mathrm{P}_{\mathrm{rf}}$. The phase dependence is close to a $v^{1 / 2}$ dependence, as will be discussed in Section 5. 


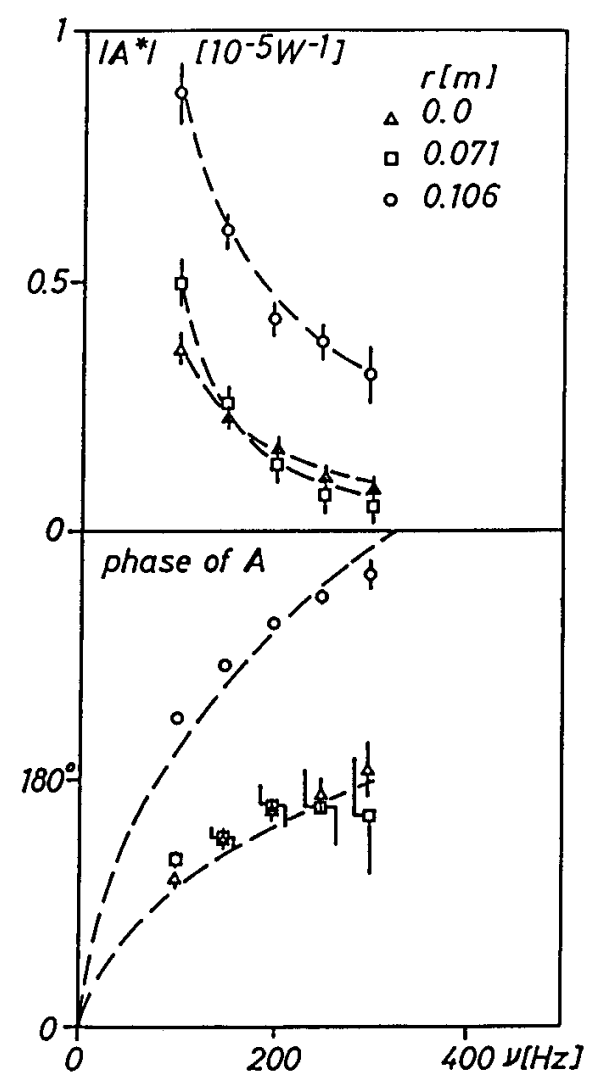

Fig. 4 Spectra of the soft $X$-ray signal response for 3 channels, $r=0.0,0.071,0.106 \mathrm{~m}$, for the data of Fig. 5.

The full radial profiles of amplitude and phase are shown for the different frequencies in Fig. 5, for one given experimental condition. There are two distinct radial zones. The central region within $\mathrm{q}=1$, out to a radius of $0.07 \mathrm{~m}$, has an almost flat phase profile, with a tendency to be slightly convex with a higher phase on axis, especially at higher frequency. Outside this region the phase is much larger, with a sharp jump between the two regions, beyond the resolution of the diode array. In this second region the phase tends to increase with radius, except at the highest frequencies $(v>300 \mathrm{~Hz})$.

In the inner region the response gain is fairly flat, less than $0.5 \% / \mathrm{kW}$ at $100 \mathrm{~Hz}$. In the outer region the modulation of the soft $\mathrm{X}$-ray flux is much stronger, up to $2 \% / \mathrm{kW}$ at the same frequency. This large relative modulation towards the edge of the plasma might be considered surprising, as we would imagine an attenuation of any modulated temperature perturbation as it travels outwards. In fact the amplitude profile resembles 


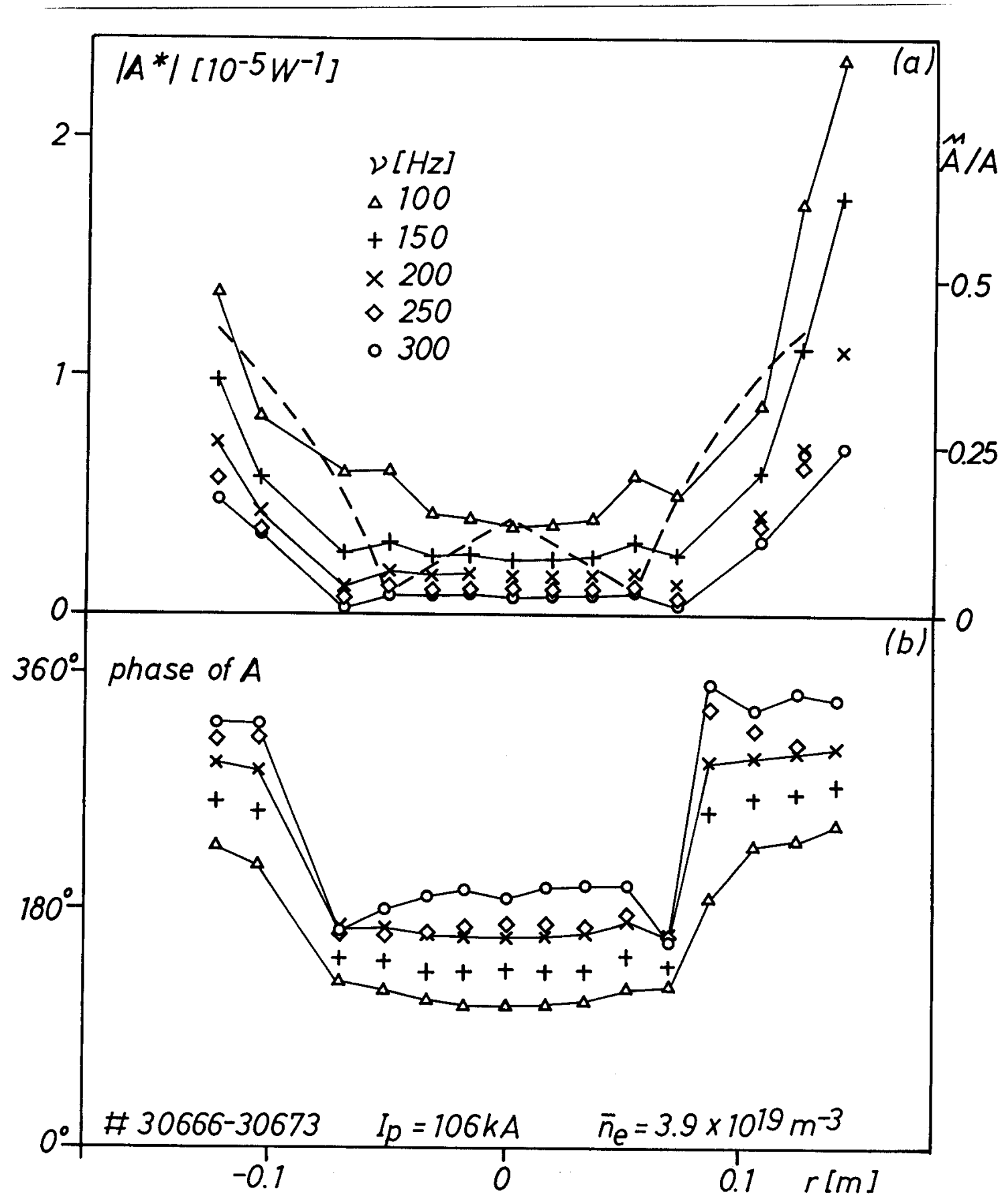

Fig. $5 \quad$ Radial profile of the amplitude (a) and phase (b) of the soft $X$-ray signal response, for different modulation frequencies between 100 and $300 \mathrm{~Hz}[2.0 \mathrm{MHz}$, $(N, M)=(2,1), \quad 105 k A, 3.9 \cdot 10^{19} \mathrm{~m}^{-3} \mathrm{~J}$. A typical relative amplitude of the sawtooth crashes in an ohmic discharge is also shown as a dotted line.

that of the sawtooth crash relative amplitude, shown in Fig. 5(a) as a dashed line. The origin of this dependence is assumed to be a mixture of the density profile effect and the 
temperature exponent $\alpha\left(T_{e}\right)$ increasing at the lower temperature towards the edge of the plasma.

The regular form of the data in Figs 4 and 5 illustrates that the measurement accuracy is adequate to describe the large variations in the gain and phase found in the experiment.

\subsection{Non-harmonic response}

It is interesting to see whether the dynamical response obtained with harmonic modulation bears any resemblance to the dynamical response to other irregular changes in the plasma. Figure 6 shows the raw data obtained under different transient conditions. In Fig. 6(c) we simply cut the RF pulse; in Fig. 6(b) the density evolution crossed the $(\mathrm{n}, \mathrm{m})=(2,0)$ Discrete Alfvén Wave eigenmode, causing a change in the predicted power

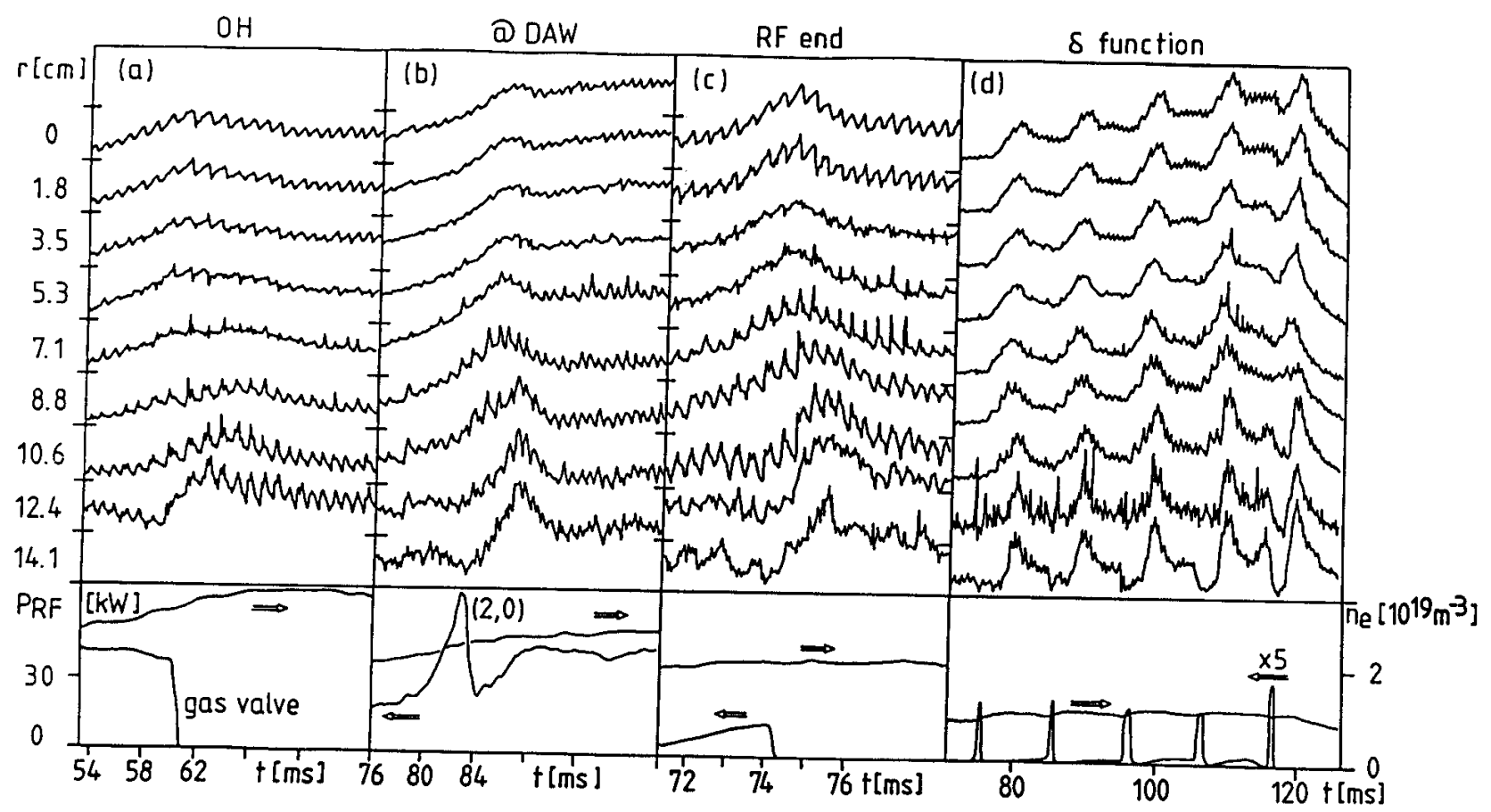

Fig. 6 Raw signal traces for discharges used for evaluating the transient response delay $[2.5 \mathrm{MHz}, 120 \mathrm{kA}$, $(N, M)=(2,1)]:(a)$ OH gas cut; (b) $(n, m)=(2,0) D A W$ crossing; (c) RF turn off; (d) $120 \mathrm{~kW}$ periodic pulses. 
deposition profile as well as a clear spike in the delivered RF power; in Fig. 6(d) we preprogrammed a series of short RF pulses and in Fig. 6(a), with no additional RF heating, the gas-valve was suddenly closed. In all these cases there is a marked response on all soft $\mathrm{X}$-ray channels, at a time varying from one diode to another. Figure 7 collates the data from these discharges, plotting the delay of a marked feature as a function of minor radius. In this case the signal maximum is used to identify the propagation. There is a striking resemblance between the delay profiles for all four transient responses and the harmonic response profile, although the times are not identical. The harmonic response delay for an outer diode at a frequency of $500 \mathrm{~Hz}$ is $2.0 \mathrm{msec}$ which is not far from the measured transient values. The same flat region with a low delay is followed by a rising delay further out. The similarity of a non-RF discharge suggests that the response may be general to any disturbance, rather than being a property of the additional heating.

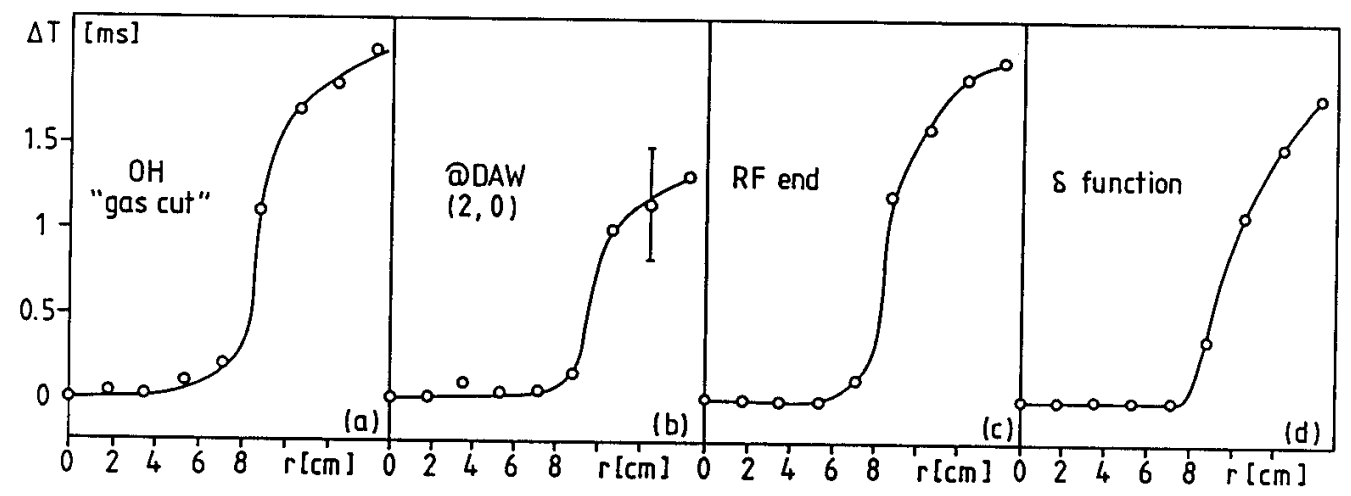

Fig. 7 Radial profile of the delay time for the transient response discharges of Fig. 6.

Figure 6(d) shows a variation in the transient response from the first pulse, due to which the outer diodes respond only after a delay, to the last pulses after which the outer diodes respond negatively immediately and positively after the same delay as before. This negative excursion, explained in harmonic response terms by the large phase of the outer diode, disappears if the spacing between pulses is increased to $20 \mathrm{msec}$, but is always present if the RF power waveform contains some continuous pedestal, as in the case of sinusoidal modulation. 
3.4 Variation of the RF excitation mode

Varying the excited Alfvén Wave mode numbers is predicted to vary the power dissipation profile, assumed to be fairly local to the Alfvén Wave resonance layers. Two attempts have been made to exploit this variation in power deposition profile.

Firstly we looked at the time-resolved harmonic response as the density evolved during the RF pulse. The increasing density causes the resonance layers to move outwards so as to continuously satisfy the resonance condition

$$
\omega^{2}(r)=\left(n+\frac{m}{q(r)}\right)^{2}\left(\frac{B_{\Phi}^{2}}{\mu_{0} \rho(r) R^{2}}\right)\left(1-\frac{\omega^{2}}{\omega_{c i}^{2}}\right)=\omega_{g e n}^{2}
$$

in which $(n, m)$ are the toroidal and poloidal mode numbers, $q(r)$ and $\rho(r)$ are the local values of safety factor and mass density. We would expect, therefore, that the phase and possibly the gain of the response would be sensitive to this evolution, at least at some minor radius. Such an experiment is shown in Fig. 8, where the temporal evolution of the phase profile is represented by a contour plot of the cosine of the phase, a value of 0 corresponding to a phase of $270^{\circ}$. Although during this discharge the density increased from $2.5 \cdot 10^{19} \mathrm{~m}^{-3}$ to $5.0 \cdot 10^{19} \mathrm{~m}^{-3}$, pushing the resonance layers outwards, no significant modification of the phase profile can be measured, especially in the upper half where the diode array resolution is better.

In principle this type of experiment should be extremely sensitive to any sudden variation, due to the inherently good resolution within one discharge.

Secondly, we varied the excitation mode structure from $(\mathrm{N}, \mathrm{M})=(2,1)$ to $(N, M)=(4,1)$, with the results shown in Fig. 9. Although the curves do show slight variation, the calculated deposition profile, which is characterised in the case of the 
$(N, M)=(4,1)$ excitation by edge localised resonance layers, is clearly not determining the fundamental form of the response curves.

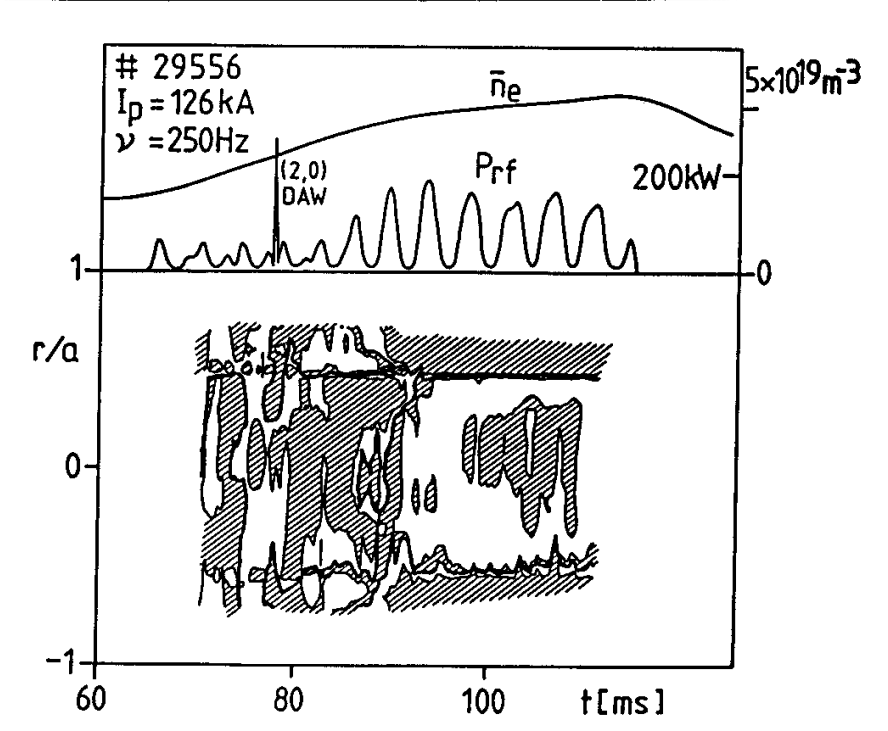

Fig. 8 Time history of the response phase when crossing the $(n, m)=(2,0)$ DAW and threshold while the density increases $[2.5 \mathrm{MHz}, 120 \mathrm{kA},(N, M)=(2,1)]$. The contours represents values of the cosine of the phase of $-0.5,0.0,0.5$; the dark line corresponds to a phase of 270 ?

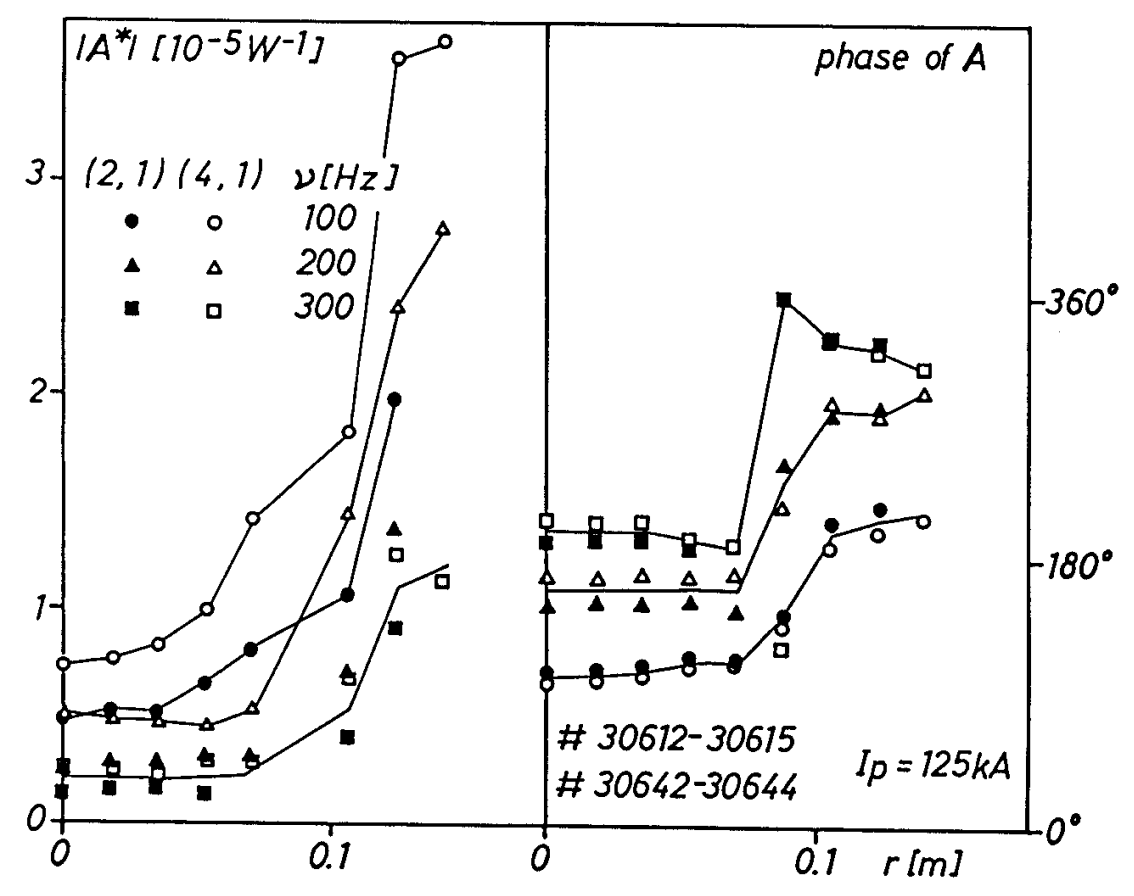

Fig. 9 Radial profile of the modulation response when the excitation condition is changed from $(N, M)=(2,1)$ to (4,1) $[2.0 \mathrm{MHz}, 120 \mathrm{kA}]$. 


\subsection{Effect of plasma current}

A plasma current scan was carried out, at fixed magnetic field, over the range of $55-121 \mathrm{kA}\left(\mathrm{q}_{\mathrm{a}}\right.$ from 6.8 to 3.2$)$. Figure 10(a,b) shows the harmonic response gain and phase for one frequency, $100 \mathrm{~Hz}$, although the experiments were performed from 100$300 \mathrm{~Hz}$ leading to identical conclusions. All curves show the same fundamental form seen in the previous sections. However, this time the scan produces a dramatic change in the size of the inner region, which shrinks as $\mathrm{q}_{\mathrm{a}}$ increases. The central current profile is considered to shrink with increasing $q_{a}$, usually well approximated by $r_{q=1} \approx a / q_{a}$. A phase contour plot in the (r, $\mathrm{I}_{\mathrm{p}}$ ) plane (Fig. 10(c)) shows that this shrinking is parallel to the sawtooth inversion radius, and is close to the $\mathrm{a} / \mathrm{q}_{\mathrm{a}}$ approximation.

Again, we have attempted to relate these observations to an arbitrary transient response, in this case the RF turn-off. Figure 11 shows delay curves, similar to those produced for Fig. 7, for three values of plasma current giving $\mathrm{q}_{\mathrm{a}}=3.1,4.0,6.8$. Again there is a shrinking of the inner region.

The general shape of the phase profile bears some resemblance to a purely diffusive curve in the presence of strongly central heating, as deduced by JAHNS et al. (1986) on D-III. However, this current scan obliges us to conclude that the general form of the response phase profile is governed by the plasma current, which has a much greater effect than the assumed deposition profile. The position of the $q=1$ surface plays the dominant role in the shape of the curve.

\subsection{Power dependence}

All the results described in the previous subsections were obtained using a roughly constant RF power modulation depth. Before we attack the question of the power dependence of the response gain and phase, we must recognize that the gain is not constant during the full RF pulse, an effect which is visible in Fig. 3. In Fig. 12 we 


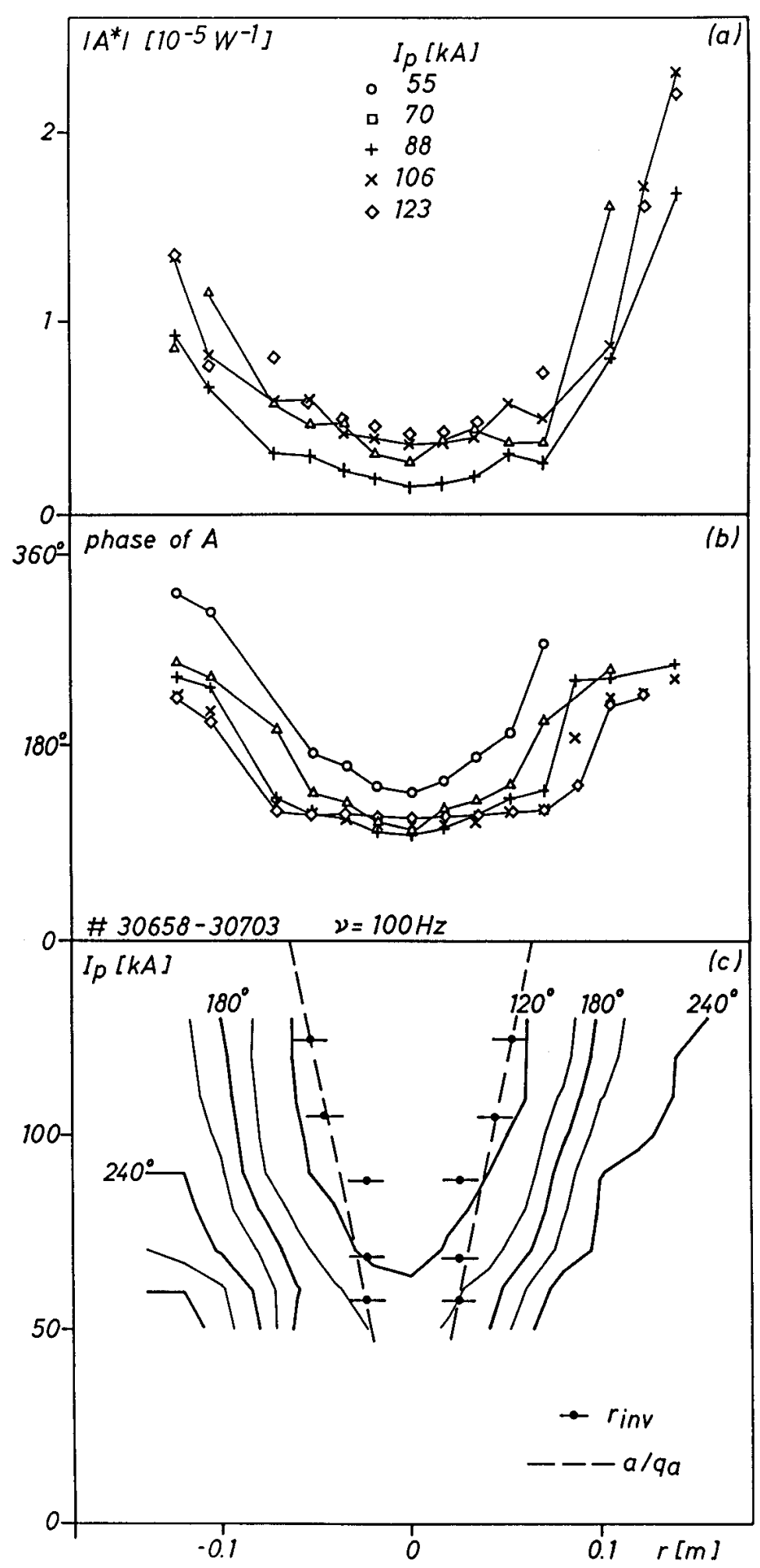

Fig. 10 Radial profile of the response amplitude (a) and phase (b) at $100 \mathrm{~Hz}$ as the plasma current is varied. The variation of the sawtooth inversion radius and the dependence $a / q_{a}$ are also shown on the contour plot (c) $[2.0 \mathrm{MHz},(N, M)=(2,1)]$. 


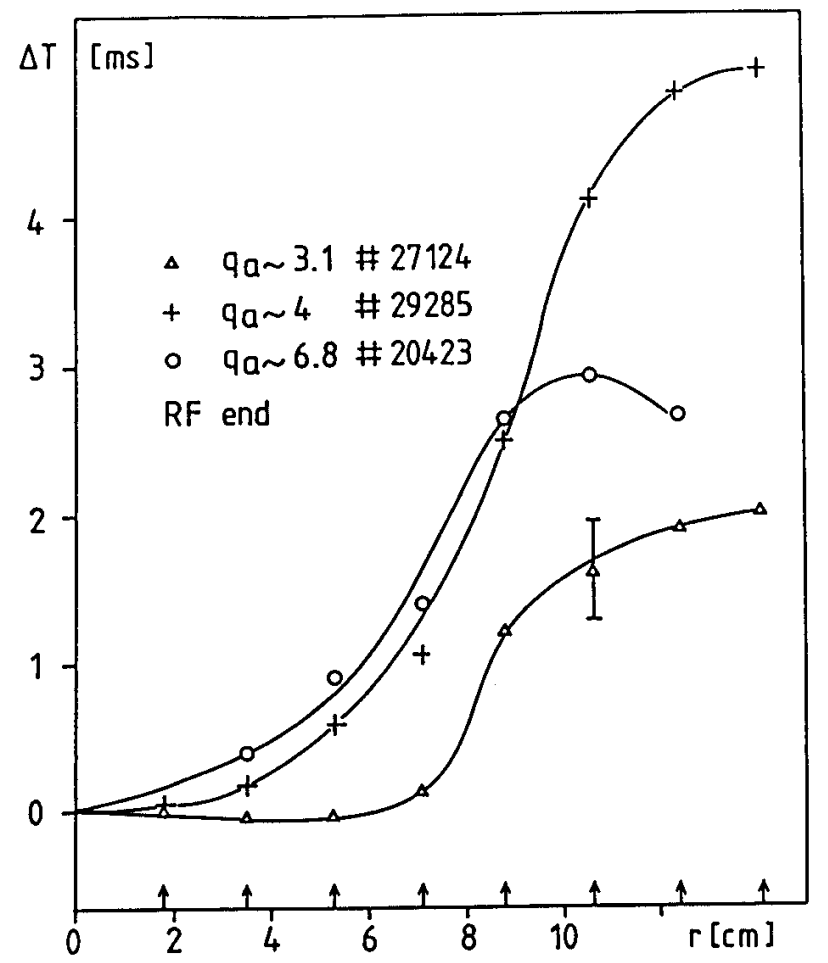

Fig. 11 Radial profile of the turn-off response delay for different values of plasma current and safety factor $[2.5 \mathrm{MHz},(N, M)=(2,1)]$.

present the harmonically modulated part of the fits to the RF power and the central chord soft X-ray signal. When the RF power is modulated from the beginning, Fig. 12(b), the ratio between the amplitudes is constant at its asymptotic value after $30 \mathrm{msec}$, as it is for the full duration of the gated modulation, Fig. 12(a). During the first $40 \mathrm{msec}$ of the fully modulated discharge the ratio increases towards the asymptotic value. Throughout the modulation the phase is constant.

Having isolated this effect, Fig. 13 presents the power dependence of the response amplitude, estimated during the last $40 \mathrm{msec}$ of an $80 \mathrm{msec}$ RF pulse with an RF power DC level of $40 \mathrm{~kW}$. It clearly demonstrates that for this power level a linear response regime is reached after $40 \mathrm{msec}$. The response of a set of discharges including higher RF power DC levels is analysed during the last $10 \mathrm{msec}$ of a $50 \mathrm{msec} \mathrm{RF}$ pulse. The gain times the radian frequency, $\omega \mid \mathrm{A} *$, for a frequency range of $100 \mathrm{to} 500 \mathrm{~Hz}$, is a decreasing function of the modulation depth, Fig. 14, showing that these discharges are not in a linear response regime. Nevertheless the phase in these conditions does not 


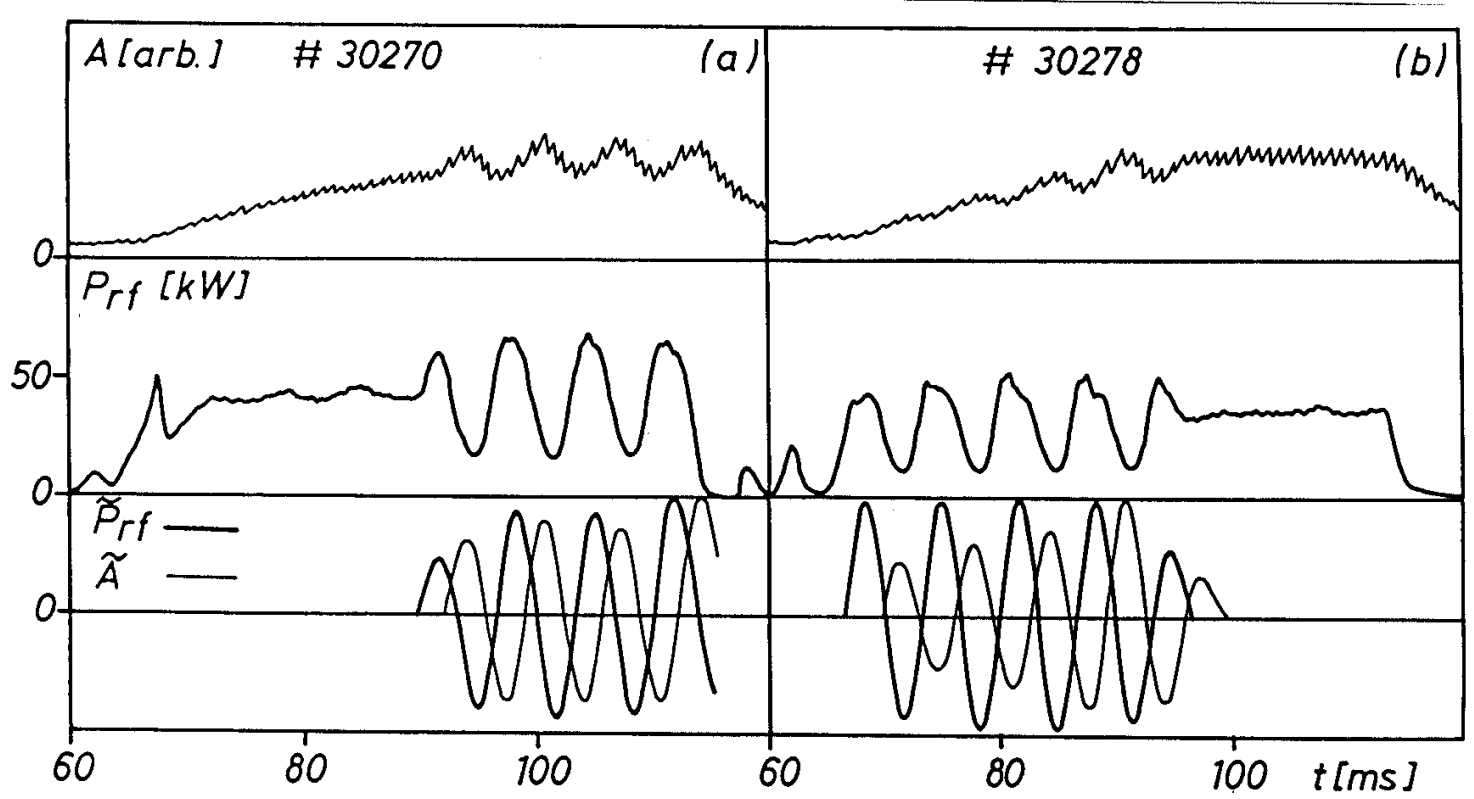

Fig. 12 Superposition of the harmonic part of the fitted forms of $P_{r f}$ and soft $X$-ray signal on axis for gated and fully modulated discharges $[2.5 \mathrm{MHz}, 120 \mathrm{kA}$, $(N, M)=(2,1)]$.

depend on the modulation depth, indicating that this non-linearity is a pure amplitude non-linearity. This result implies that when the RF power is turned on, the plasma requires a minimum time with RF before it can respond linearly in amplitude to the power modulation, although the phase is immediately established. This may be equivalent to a limit on the slew rate of a plasma parameter when we superimpose the low frequency turn-on response to the RF pulse onto the modulation. Comparing Figs. 12(a,b), it is clear that the turn-on of the RF pulse is responsible, and not the start of the modulation.

\section{MODULATION OF THE SAWTEETH}

Changes in the sawtooth period have already been noted during AWH experiments on TCA, and were related to changes in the deposition profile consequent to changes in the excited spectrum (BESSON et al., 1986). The modulation experiments described in the preceding section also systematically produced a periodic modulation of the sawtooth frequency $1 / \tau_{\mathrm{ST}}$ as well as the sawtooth slope $\mathrm{dA}_{\mathrm{ST}} / \mathrm{dt}$ (Fig. 3). The sawtooth period is significantly modified, from $0.8-1.4 \mathrm{msec}$ in the examples shown with a $150 \mathrm{~Hz}$ modula- 


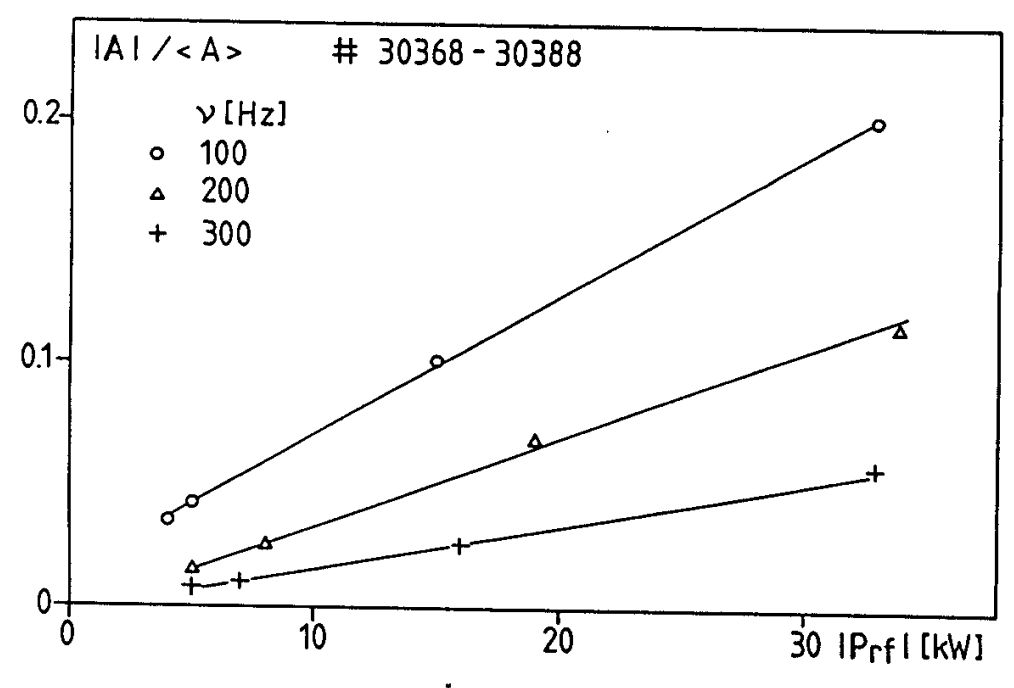

Fig. 13 Relative modulation amplitude as a function of the modulation depth for an RF power DC level of $40 \mathrm{~kW}$ $[2.5 \mathrm{MHz}, 120 \mathrm{kA},(N, M)=(2,1)]$.

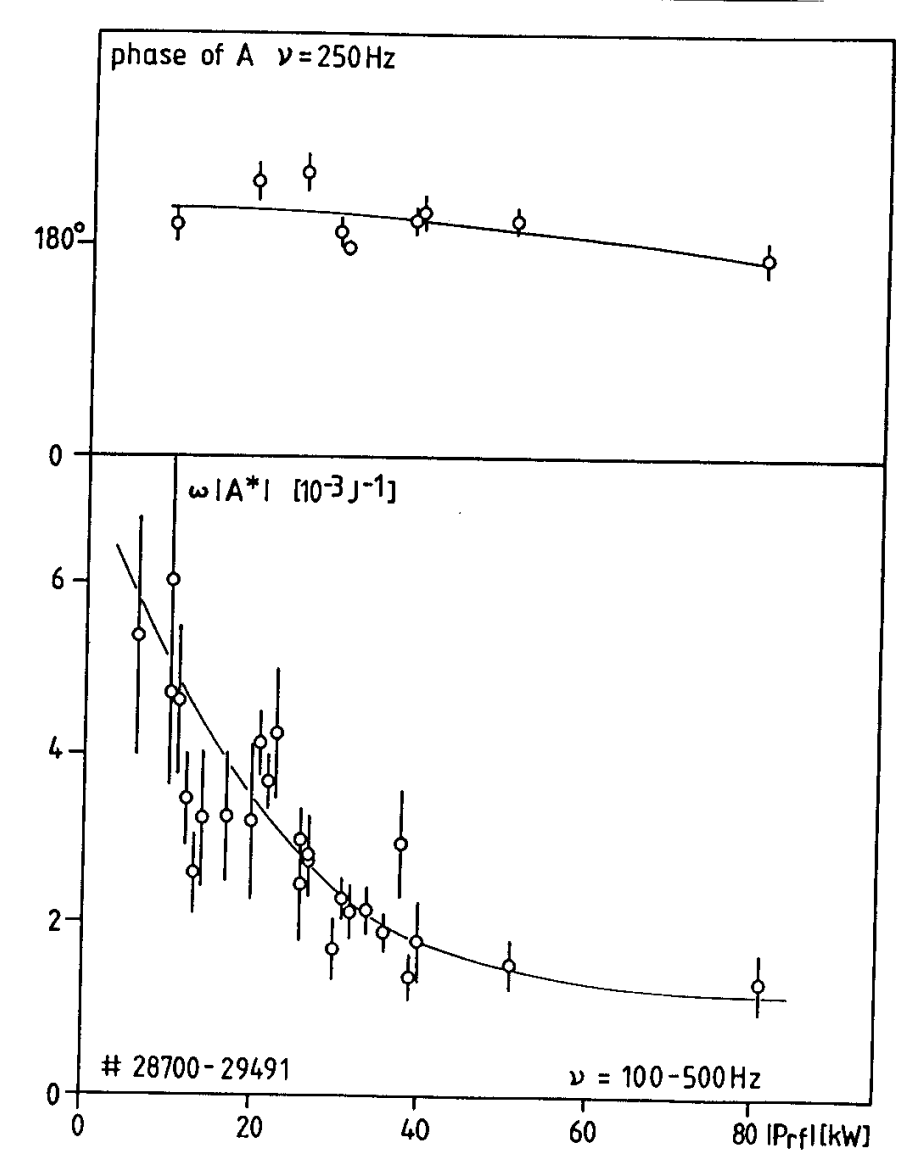

Fig. 14 Power dependence of the response gain times the radian frequency and of the phase at $250 \mathrm{~Hz}$ for different modulation frequencies $[2.5 \mathrm{MHz}, 66$ $125 \mathrm{kA},(N, M)=(2,1)]$. 
tion frequency. The periodic modulation is clearly not in phase with the RF power, and the relationship between the two is most apparent on a Lissajou plot of period versus RF power, for several modulation frequencies and amplitudes, Fig. 15. The

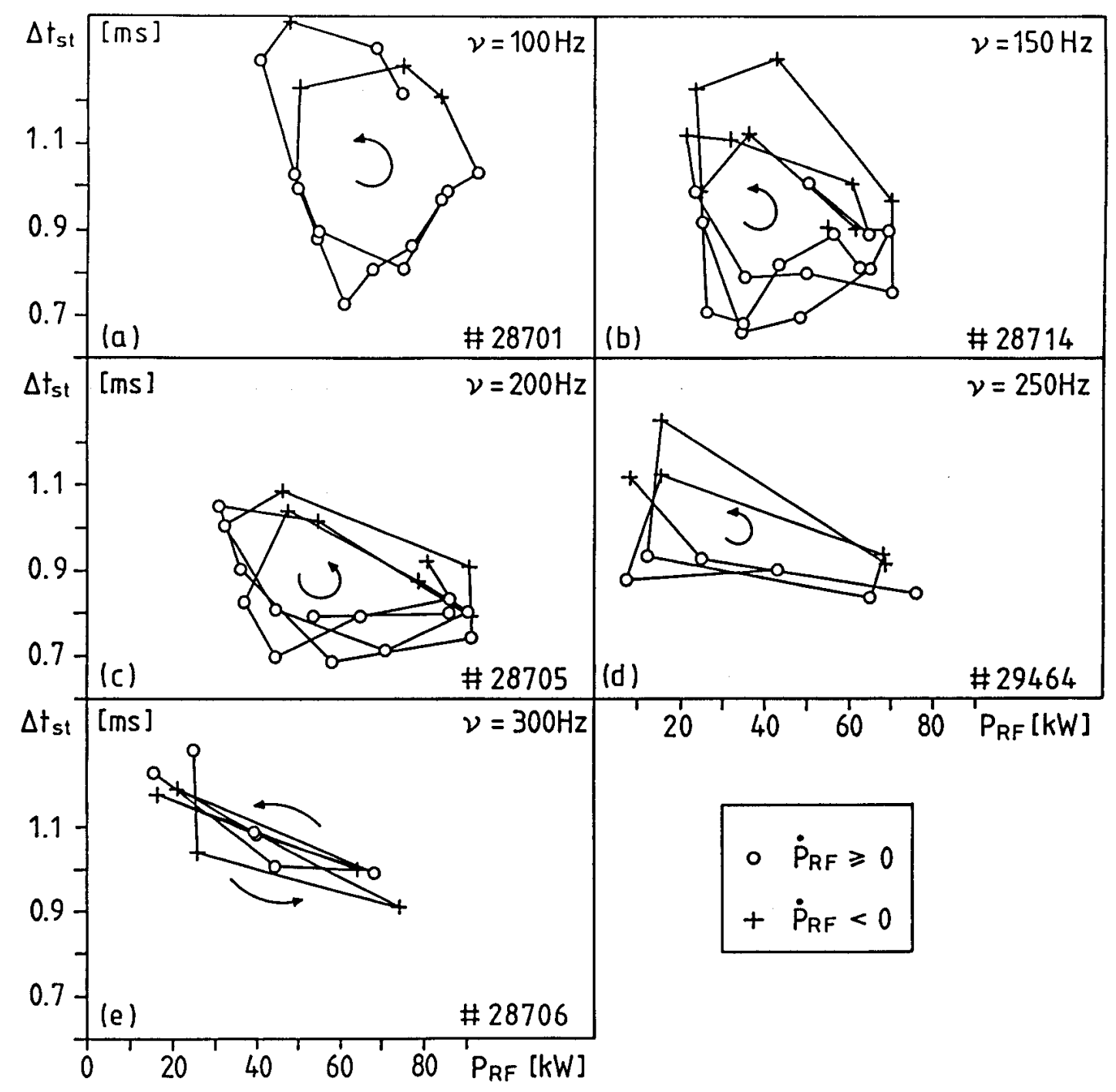

Fig. 15 Lissajou plots of the sawtooth period modulation and the $R F$ power modulation, for different powers and frequencies $[2.5 \mathrm{MHz}, 120 \mathrm{kA},(\mathrm{N}, \mathrm{M})=(2,1)]$.

relative phase close to $90^{\circ}$ implies that the RF power is not the direct cause, but that its integral causes an increase in sawtooth period. Since this phase-shift is strong at a modulation frequency of $100 \mathrm{~Hz}$, its integration time constant must be longer than the sawtooth period. At low frequency, less than $50 \mathrm{~Hz}$, we recover a tracking of the density dependence of the sawtooth period, with no hysteresis in the Lissajou plot. 
The variation of the sawtooth period initially increases with the modulated RF power but is fairly constant above $40 \mathrm{~kW} \mathrm{p-p.} \mathrm{As} \mathrm{the} \mathrm{frequency} \mathrm{is} \mathrm{increased} \mathrm{the} \mathrm{upper}$ and lower period bounds converge towards the unmodulated period (Fig. 16). The RF

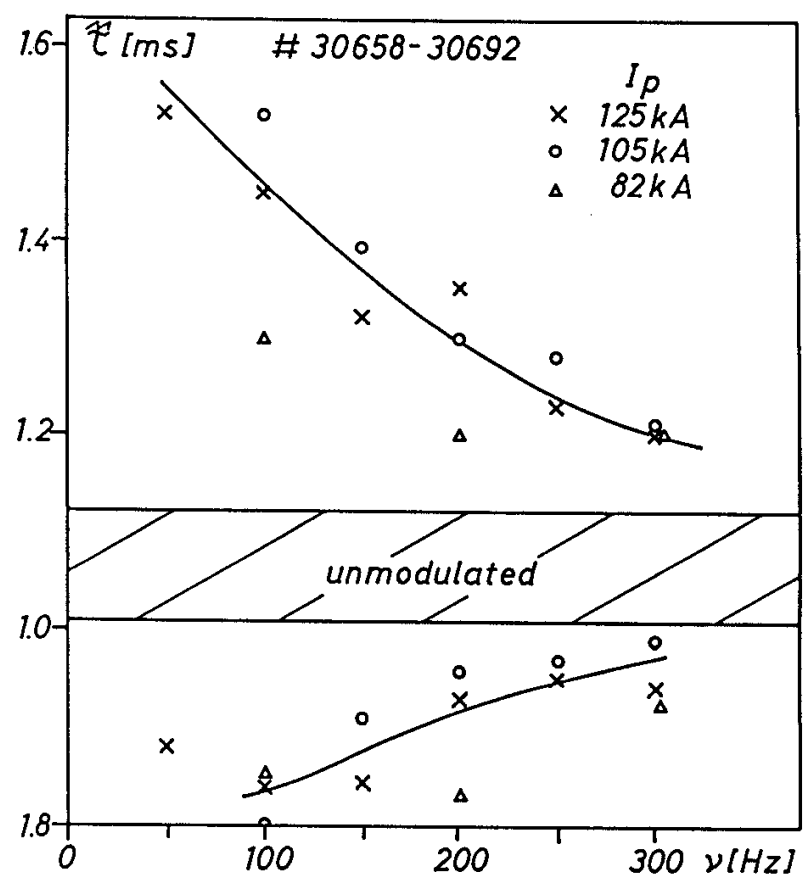

Fig. 16 Upper and lower limits of the modulated sawtooth period as a function of modulation frequency and plasma current $[2.0 \mathrm{MHz},(N, M)=(2,1)]$.

power modulation is therefore producing both an increase and a decrease with respect to the unmodulated case. At lower plasma current the variation is smaller, being unmeasureable below $I_{p}=89 k A\left(q_{a} \approx 5\right)$ due to noise on the estimate of the sawtooth period.

At first it was considered that the effect of the RF power modulation might be analogous to results obtained by GAMBIER (1987) on JET. In the results presented here, however, we have shown that the period modulation is sinusoidal with a frequency much lower than the sawtooth frequency, and cannot be attributed to a statistical explanation. Similarly, the phase lag shows that the instantaneous RF power is not, in our case, responsible for the modulation. This has been confirmed by modulating the RF power rapidly compared with the sawtooth frequency ( $v$ up to $3 \mathrm{kHz}$ ), in which case no effect on the crash probability was detected. 
The change in period is not caused by the density modulation, nor can it be simply attributed to a modulation of the $\mathrm{q}=1$ radius, as the sawtooth inversion radius remains constant within the statistical variation $( \pm 2 \mathrm{~mm})$. In the absence of a clearly accepted sawtooth model, it is difficult to propose a mechanism by which the period is modulated.

The hysteresis in Fig. 15 has several implications, however. Firstly, since the hysteresis is a function of frequency, the evolution of the sawtooth period will depend upon the rate at which the experimental conditions evolve. The large change in sawtooth period observed when crossing an Alfvén Wave eigenmode, or threshold, was in fact much less marked when the experiment was repeated very slowly in more "controlled" conditions! It is clear that we cannot ever succeed in stabilising the spectral conditions in the long sawtooth period region, since this is an inherently transient or dynamical phenomenon. Secondly, the result has an importance in interpreting the deposition power in terms of the central value of $\mathrm{dW}_{\mathrm{e}}(0) / \mathrm{dt}$ obtained from the sawtooth ramp (JOYE et al., 1986). Although the energy derivative has to come from somewhere, the fact that it is not in phase with the applied RF modulation puts a question mark on the interpretation as evidence of a direct power transfer from the wave. The steady state results reported by Joye et al. could not show up this discrepancy, illustrating the extent to which the harmonic modulation provides extra information.

\section{DISCUSSION}

In this section we preliminarily interpret the phase and amplitude profiles obtained in Section 3 from the harmonic modulation experiments. In slab geometry, with a given localised power source, and with $n_{e}$ and $\chi_{e}$ constant, the diffusivity leads to a travelling temperature perturbation whose phase delay in the region where there is no power deposition will be proportional to $\left(v / \chi_{\mathrm{e}}\right)^{1 / 2} \Delta \mathrm{x}$, where $\chi_{\mathrm{e}}$ is the thermal conductivity and $\Delta \mathrm{x}$ is the distance from the localised source. The phase at the source, where the time derivative of the temperature is directly related to the power, will be a maximum of $90^{\circ}$ at the highest frequency. Figure 4 shows a $v^{1 / 2}$ dependence superimposed on the phase spectra for the three radii, and the agreement is generally good except near the minimum phase point where the frequency dependence of $\left|A^{*}(v)\right|$ is stronger than $\mathbf{v}^{-1}$. In cylindrical 
geometry the $v^{1 / 2}$ dependence is retained, whereas the radial variation will depart from the proportionality in $\Delta \mathrm{x}$ of the simple mentioned slab relation. Nonetheless we can deduce a lower limit on $\chi_{e}$ in the central region of flattish phase, yielding $\chi_{e}>5 \mathrm{~m}^{2} / \mathrm{s}$. This large value is in agreement with our understanding of transport within the $q=1$ radius being very rapid, dominated by the sawtooth activity. The large jump in phase at the $\mathrm{q}=1$ radius must, in a simple model, correspond to a region of higher thermal insulation.

The full information is, however, more enigmatic than this simple picture. Some of the data support a simple deposition and diffusion model, and some contradict such a model. The $v^{1 / 2}$ diffusive nature of the phase spectrum at all radii would suggest the presence of a local source away from all these measurement radii. The existence of such a local source will also automatically imply the presence of a local maximum of $\left|\mathbf{T}_{e}\right| /\left\langle T_{e}\right\rangle$, provided that the modulation frequency is greater than a critical frequency proportional to $1 / \chi_{\mathrm{e}}$, which should be satisfied in our experiments at the modulation frequencies used. There are in fact indications that such a maximum may exist inside, but always near the edges of the inner region (Fig. 5). This radial position also corresponds to the radius of minimum phase which is an essential requirement for the localised deposition plus diffusion model. However, two problems remain. Firstly the minimum phase radius is governed by the value of $\mathrm{q}_{\mathrm{a}}$ and not by the assumed physics of Alfvén Wave Heating. Secondly, the minimum phase is much too high. In Fig. 4, the minimum phase at maximum frequency is never less than $155^{\circ}$, incompatible with the $90^{\circ}$ permitted by a local integration of the locally deposited power. The errors in the measurements could not total more than $35^{\circ}$ in the worst imaginable case.

One possible explanation for this result is that there is in fact a point of minimum phase, at $90^{\circ}$, but that it is extremely narrow in radial extent. It could not, therefore, be measured with our soft $X$-ray array with its $18 \mathrm{~mm}$ resolution at that radius. The rapid phase jumps inside and outside the minimum phase radius would imply a very narrow region of high thermal insulation on both sides of the source radius, where $q=1$, for all observed conditions. This argument leads to a result which is strangely similar to that of the TFR GROUP (1987) with pellet injection, in which the ingoing cold front suddenly stopped at the $q=1$ surface. Similarly the LHRH experiments on ASDEX showed that the 
electron heating was limited to within the $\mathrm{q}=1$ radius (STEINMETZ et al., 1987). At low plasma current the $\mathrm{q}=2$ surface is also well within our observed radii, although no such discontinuity has ever been seen associated with it. Instead of being spatially averaged out, a narrow minimum phase region might be temporally smoothed out if its position varied, or if it rotated as a rigid structure.

Alternatively, if we accept that the minimum observed phase is correct, then we could explain the delay in terms of an additional mechanism creating a second order system with another time constant. In this case this indirect mechanism equivalently absorbs and releases the RF energy, allowing the minimum phase to increase to $180^{\circ}$ at high frequency. In the case of minority heating (JET TEAM, 1987) such an intermediate mechanism was postulated to generate the $\mathrm{v}^{-2}$ frequency dependence of the response amplitude, although no data were given on the phase. In the case of Alfvén Wave Heating, the wave damping rate by Electron Landau Damping could not generate a significant delay, nor could the thermalisation of the slightly non-Maxwellian parallel driven electrons. Transport via the ions would provide an ideal candidate, but electronion power flow is relatively insensitive to the ion temperature in our conditions and the measured ion temperature modulation is small.

A possible hypothesis is that part of the RF energy is stored as a current profile modification which can release its energy to the electrons. Current diffusion provides a long time-constant integration, allowing an extra $90^{\circ}$ phase to be added. A modulation of $10 \%$ in $\beta$ would need of the order of $10 \%$ modulation in $\mathrm{l}_{\mathrm{i}} / 2$. An advantage of this hypothesis is that the current profile change might somehow be able to release its energy predominantly at the $\mathrm{q}=1$ radius, in agreement with our data. The role of the $\mathrm{q}=1$ surface is then to provide a local additional power "acceptance" point from which any electron temperature profile modification must originate. This hypothesis would also be supported by other experimental evidence on TCA which indicates a change in $l_{i}$ during the RF pulse (BESSON et al., 1987). It might also suggest a link with the modulation of the sawtooth period. 
To summarize, we find that the diffusive form of the phase spectrum requires that one of the following models must apply :

either (a) the RF energy is accepted in phase with the RF power, over too fine a radial extent to be seen, and at a radius which is given by $\mathrm{q}=1$ and not by the Alfvén Wave resonance condition. The local thermal conductivity on both sides is so low that the phase increases by $90^{\circ}$ to $180^{\circ}$ within the spatial resolution;

or (b) the energy deposition occurs close to the $\mathrm{q}=1$ surface, independently of the Alfvén Wave resonance condition, but is delayed by a higher order element in the system causing a minimum phase near $155^{\circ}$.

\section{SUMMARY}

The wide experimental range of modulation frequency and plasma current used in these experiments has brought out new features to modulation experiments. The form of both the amplitude and phase profiles is dominated by the role of the $\mathrm{q}=1$ surface during Alfvén Wave Heating. The phase in the centre of the plasma is larger than a simple model would predict, and the minimum phase, near $\mathrm{q}=1$, is just less than $180^{\circ}$. Simply examining the response amplitude would not have highlighted the discrepancies. Two types of explanation have been discussed, without being able to confirm either of them. The sawtooth period is modulated by the RF power, but $90^{\circ}$ out of phase. Since previous modulation experiments have not explored the extra information available in the relative phase, we cannot state whether these observations are peculiar to Alfvén Wave Heating or are more general.

Acknowledgement - We are grateful for the assistance of the whole TCA team and the interest of Prof. F. Troyon. The work was partly funded by the Fonds National Suisse de la Recherche Scientifique. 


\section{REFERENCES}

BESSON G., DE CHAMBRIER A., COLLINS G.A., JOYE B., LIETTI A., LISTER J.B., MORET J.-M., NOWAK S., SIMM C. and WEISEN H. (1986) Plasma Phys. and Contr. Fusion 28, 1291.

CHEETHAM A.D., HEYM A., HOFMANN F., HRUSKA K., KELLER R., LIETTI A., LISTER J.B., POCHELON A., RIPPER H., SIMIK A., and TUSZEL A. (1981) Proc. 11th Symp. Fusion Techn. 1, 601.

COLLINS G.A., HOFMANN F., JOYE B., KELLER R., LIETTI A., LISTER J.B., and POCHELON A. (1986) Phys. Fluids 22, 2260.

GAMBIER D. (1987) Private communication.

HARTFUSS H.J., MAASBERG H., TUTTER M., WVII-A GROUP and ECRH GROUP (1986), Nucl Fusion 26, 678.

JAHNS G.L. WONG S.J. PRATER R., LIN S.H. and EJIMA S. (1986) Nucl. Fusion 26, 226.

JET TEAM (1987) XI IAEA Conf. on Plasma Phys. and Contr. Nucl. Fusion Research (Kyoto, Japan) I, 449.

JOYE B., LIETTI A., LISTER J.B., MORET J.-M. and SIMM W. (1986) Phys. Rev. Lett. 56, 2481.

STEINMETZ K., SOELDNER F.X., ECKHARTT D., JANESCHITZ G. et al. (1987), XI IAEA Conf. on Plasma Phys. and Contr. Nucl. Fus. Research (Kyoto, Japan) I, 461.

TFR GROUP (1987) 14th EPS Conf. Contr. Fus. Plasma Phys. (Madrid) $1,29$. 